\title{
Médiévales
}

Langues, Textes, Histoire

55 | automne 2008

Usages de la Bible

\section{Usages de la Bible et institution du sens dans l'Occident médiéval}

\section{Michel Lauwers}

\section{(2) OpenEdition \\ 1 Journals}

Édition électronique

URL : https://journals.openedition.org/medievales/5436

DOI : $10.4000 /$ medievales. 5436

ISSN : $1777-5892$

Éditeur

Presses universitaires de Vincennes

\section{Édition imprimée}

Date de publication : 20 décembre 2008

Pagination : 5-18

ISBN : 978-2-84292-221-4

ISSN : 0751-2708

\section{Référence électronique}

Michel Lauwers, «Usages de la Bible et institution du sens dans l'Occident médiéval », Médiévales [En ligne], 55 | automne 2008, mis en ligne le 20 mars 2011, consulté le 21 septembre 2021. URL : http:// journals.openedition.org/medievales/5436; DOI : https://doi.org/10.4000/medievales.5436

Ce document a été généré automatiquement le 21 septembre 2021.

Tous droits réservés 


\title{
Usages de la Bible et institution du sens dans l'Occident médiéval
}

\author{
Michel Lauwers
}

1 La Bible était au Moyen Âge indissociable de ses commentaires, et ceux-ci ont été un lieu de réflexion et d'élaborations conceptuelles propres à rendre intelligible, à maitriser ou à transformer la société. Partant de telles prémisses, les chercheurs réunis à Auxerre, le 24 janvier 2008, pour préparer ce dossier de la revue Médiévales se sont attachés à quelques cas d'interprétation du texte sacré à l'évidence marqués par les besoins de leur temps, ainsi qu'aux principes d'herméneutique qui ont pu guider les acteurs sociaux ou légitimer leur action ${ }^{1}$.

2 La question des usages de la Bible en Occident chrétien doit être rapportée à deux données structurelles. La première concerne la place très particulière du Livre sacré dans les systèmes de normes en vigueur dans la société médiévale. La valeur prescriptive de la Bible fut, en effet, bien moindre dans le monde chrétien que ne l'était celle de la Torah pour les Juifs ou celle du Coran dans les pays musulmans ${ }^{2}$ : l'existence dans l'Occident chrétien d'un autre corpus de règles, le droit romain, explique en partie cette situation. Celle-ci ne concerne toutefois que l'Antiquité tardive, un monde où le droit organisait une part importante des rapports sociaux, puis l'ère qui s'ouvrit aux $\mathrm{XII}^{\mathrm{e}}$ et $\mathrm{XIII}^{\mathrm{e}}$ siècles, lorsque le processus de genèse de l'état imposa dans les pays européens un nouveau développement des savoirs et des institutions juridiques. Entre ces deux moments, la Bible fut pendant plusieurs siècles l'une des principales sources de l'autorité. Illustrant la fonction normative alors reconnue au texte sacré (et à son commentaire), le catalogue de la bibliothèque de Reichenau, l'une des grandes abbayes carolingiennes, signale, sous une rubrique annonçant des livres de «lois»(de legibus), un ouvrage réunissant «les capitulaires de l'empereur Charles et les gloses sur la Genèse, l'Exode, le Lévitique, les Nombres, le Deutéronome, les livres des Juges, de Josué, de Ruth et des Rois ${ }^{3}$ " : ainsi le recueil des textes législatifs émanant de Charlemagne se trouvait-il associé, au IX $x^{\mathrm{e}}$ siècle, à plusieurs livres glosés de l'Ancien Testament, dans un volume rangé au sein d'une série d'ouvrages relatifs aux «lois». De manière générale, un certain nombre de règles 
énoncées dans les livres vétéro-testamentaires furent transformées, dans le haut Moyen Âge, en normes chrétiennes, diffusées par la littérature canonique, mais aussi par la législation royale ${ }^{4}$. Jusqu'au XII ${ }^{\mathrm{e}}$ siècle, les autorités (ecclésiastiques) affirmèrent d'ailleurs la dimension juridique de la Bible ${ }^{5}$. Il arriva cependant que des recommandations bibliques fussent jugées obsolètes, entraînant dans ce cas des discussions à propos des relations entre l'ancienne et la nouvelle Loi ${ }^{6}$. Soucieux de concilier l'autorité de la Bible et la pratique judiciaire de son temps, le grand canoniste Gratien entreprit, au milieu du $\mathrm{xII}^{\mathrm{e}}$ siècle, d'opérer une distinction, parmi les commandements du texte sacré, entre les moralia, auxquels devait se conformer le comportement des fidèles, et les mystica, dont le sens était allégorique ${ }^{7}$. La réception ou le dépassement de la norme biblique nécessitait de toute manière une interprétation autorisée du Livre sacré, c'est-à-dire un détour par l'exégèse. Celle-ci fut du reste un enjeu important, à partir de la seconde moitié du XII siècle, dans les débats entre théologiens et juristes.

3 L'histoire de la Bible médiévale présente une seconde particularité, qui ne fut pas sans incidence sur la structure des sociétés occidentales: si l'Écriture Sainte constituait le Livre par excellence, source (ou miroir) de tous les savoirs ${ }^{8}$, celui-ci n'était pas à la portée de tous les fidèles. L'accès au texte sacré et son interprétation furent même régulés, en Occident, par une institution spécialisée, sans équivalent dans les mondes juif et musulman. Non que la lecture et le commentaire de la Bible firent à proprement parler l'objet d'un monopole ${ }^{9}$, mais la mise en place d'un corps de ministres du sacré maîtrisant une pratique de l'écriture que bien peu désormais dominaient fit de ces spécialistes, tout entiers voués à la reproduction spirituelle de la société, des médiateurs quasiment obligés pour accéder au Livre. Ce type d'organisation n'est pas exceptionnel : comme l'écrit Jack Goody, le prêtre a toujours, dans les religions de tradition écrite, un accès privilégié aux textes sacrés, dont il est le principal interprète ${ }^{10}$. Mais, dans l'Occident médiéval, cette tendance fut accentuée au point que les notions de "clerc» (clericus) et de «lettré » ( litteratus) vinrent à se confondre. Le mot litterae (au pluriel) désignait aussi bien les lettres de l'alphabet que le texte destiné à être lu, notamment les litterae diuinae, c'est-à-dire l'Écriture Sainte. Augustin n'avait-il pas écrit que «celui qui enseigne comment comprendre l'Écriture est semblable à celui qui enseigne les lettres, c'est-à-dire le maître qui apprend à lire ${ }^{11} »$ ? Les formes prises par l'étude et la diffusion du texte sacré participaient donc à l'instauration d'un ordre social fondé sur un partage entre les clercs litterati et les laïcs illiterati.

4 La Bible et ses usages ont été, dans les dernières décennies, au cœur de maints travaux. Mis à part un article pionnier, sans postérité véritable, de Marc Bloch sur « la vie d'outretombe du roi Salomon " (1925) ${ }^{12}$, c'est à partir des années 1960 que des recherches, réalisées notamment dans le domaine de l'ecclésiologie médiévale par des historiens anglo-saxons et italiens, ont porté sur les exploitations « politiques » de la Bible ${ }^{13}$. Walter Ullmann s'est ainsi intéressé à la part prise par les modèles scripturaires dans la réflexion des clercs sur le gouvernement ${ }^{14}$. Dans le cadre de travaux sur la symbolique du pouvoir, des chercheurs se sont en particulier penchés sur les usages de certaines figures bibliques, comme celles des rois de l'Ancien Testament, érigés dès le haut Moyen Âge en modèles de comportement ou utilisés comme instruments de légitimation ${ }^{15}$. La pratique de la commande et parfois de la lecture des commentaires bibliques par les souverains carolingiens a été mise en évidence, de même que l'intérêt manifesté par les gouvernants pour les questions exégétiques ${ }^{16}$. C'est que l'autorité biblique constituait un auxiliaire 
indispensable pour comprendre, ordonner ou réformer la société ${ }^{17}$. Ainsi que l'a récemment souligné Scott DeGregorio, le thème de la réforme traversait déjà les commentaires du moine anglo-saxon Bède (m. 735), qui lisait les épisodes bibliques de la restauration de Jérusalem et de son Temple, après l'exil à Babylone, comme une sorte de guide destiné à la réorganisation de l'Église de son temps ${ }^{18}$. Si l'élaboration de modèles sociaux fondés sur une interprétation du texte biblique est attestée précocement ${ }^{19}$, ce type d'exploitation de la Bible s'accrut au IX ${ }^{\mathrm{e}}$ siècle, en raison d'une identification forte de la société à l'Ecclesia ${ }^{20}$. C'est alors qu'Haymon d'Auxerre élabora, dans ses commentaires sur l'épître aux Romains et sur l'Apocalypse, l'image d'une Église organisée en trois " ordres », prêtres, guerriers et travailleurs ${ }^{21}$. De telles utilisations du texte biblique ne concernèrent pas que le haut Moyen Âge. Les recherches de Guy Lobrichon et de Philippe Buc sur les gloses bibliques élaborées à Laon entre la fin $\mathrm{du} \mathrm{XI}^{\mathrm{e}}$ et le début du XII ${ }^{\mathrm{e}}$ siècle, puis à Paris aux XII ${ }^{e}$ et $\mathrm{XIII}^{\mathrm{e}}$ siècles, ont bien montré la façon dont les clercs débattaient alors des questions de l'ordre social, de la domination et du pouvoir, en s'appuyant sur les métaphores et les catégories que leur inspirait la lecture du texte sacré et en proposant des solutions variées, parfois contrastées, au problème de l'origine et de la légitimité du pouvoir terrestre ${ }^{22}$. Les débats et les querelles liés à la réforme grégorienne, et plus largement aux réformes des $\mathrm{x}^{\mathrm{e}}$ et $\mathrm{XI}^{\mathrm{e}}$ siècles, qui prirent parfois la forme de « combats de citations ", avaient favorisé de nombreuses manipulations, mais sans doute aussi une plus ample diffusion du texte sacré ${ }^{23}$. Au XIII ${ }^{e}$ siècle, les principes de fonctionnement de l'enseignement universitaire et les spécialisations intellectuelles qu'il cristallisa accentuèrent encore la dimension « agonistique » du recours à la Bible. Par ailleurs, d'un point de vue technique, le passage des commentaires linéaires, verset par verset, au système des gloses, plus ramassées, courant entre les lignes et les marges du texte scripturaire, participa à la publicité nouvelle du texte biblique et de son interprétation ${ }^{24}$.

5 Les contributions de Sumi Shimahara, Isabelle Rosé et Emmanuel Bain à ce numéro de Médiévales s'inscrivent dans le prolongement des recherches qui viennent d'être évoquées. Tandis que S. Shimahara propose une réflexion sur la lecture " politique » des prophéties vétéro-testamentaires à l'époque carolingienne, l'exploration du texte sacré aidant les clercs $d u$ IX $\mathrm{x}^{\mathrm{e}}$ siècle à décrypter la signification des événements qu'ils vivaient, les études d'I. Rosé et E. Bain présentent l'intérêt de suivre sur une longue durée, c'est-àdire dans différents contextes et en divers milieux, les interprétations et utilisations de deux épisodes du Nouveau Testament. Retraçant les étapes successives du travail de décryptage collectif du texte sacré auquel se livrèrent les auteurs chrétiens, I. Rosé et E. Bain y distinguent des moments-clés et des inflexions, fort similaires dans les deux cas 25 .

6 Si l'accumulation ou l'empilement des interprétations autorisées aboutit à la formation d'une tradition exégétique, qu'assumèrent toujours les clercs du Moyen Âge, ce sont bien évidemment les ruptures, les écarts et les déplacements à l'intérieur de cette tradition qui revêtent aux yeux de l'historien une forte signification ${ }^{26}$. La relation à l'autorité et le poids respectif de ce qu'Emmanuel Bain nomme le commentaire du texte biblique (qui s'inscrit dans la tradition exégétique) et ses usages sociaux (soit les sollicitations du texte sacré en fonction des besoins d'une société ou d'un groupe) connurent au fil des siècles de notables variations. Ces variations paraissent renvoyer en dernière instance aux systèmes d'autorité en vigueur et à la place occupée par l'institution ecclésiale au sein de la société. Parce qu'ils furent l'occasion pour les clercs d'aborder la question des biens ecclésiastiques et celle des lieux sacrés, les deux passages du Nouveau Testament retenus 
par I. Rosé et E. Bain s'avèrent particulièrement pertinents pour apprécier les enjeux sociaux de l'exégèse biblique et les aménagements de la tradition qu'ils appelaient parfois. Dans cette perspective, il conviendrait d'envisager avec précision les rapports complexes qu'entretenaient le genre du texte biblique commenté (un texte prophétique diffère assurément d'un livre historique de l'Ancien Testament ou d'une parabole évangélique), le type de modèle ou de représentation proposé, et la forme discursive dans laquelle se coule l'interprétation (exégétique ou non). Le dernier élément parait essentiel : y aurait-il une spécificité des interprétations s'inscrivant dans un strict cadre exégétique (commentaire ou glose) par rapport aux autres formes de sollicitation, plus diffuses, du texte sacré ? La question se pose de manière particulièrement vive à partir du moment où l'exégèse « envahit » toute forme de production écrite ${ }^{27}$.

En considérant les fluctuations des commentaires et des gloses bibliques, on ferait volontiers de la Bible médiévale une "autorité au nez de cire", que l'on pouvait " infléchir en différents sens », comme l'écrivait Alain de Lille (m. 1203) ${ }^{28}$, solliciter et manipuler au gré des besoins. Un tel jugement (de valeur) n'est cependant pas la meilleure voie pour apprécier les enjeux et les implications de l'activité exégétique, qui pose de manière cruciale la question du sens ou, plus exactement, de la production du sens dans l'Occident médiéval ${ }^{29}$.

Aborder correctement cette question impose en premier lieu de s'interroger sur l'association très étroite, attestée dès l'Antiquité, puis tout au long du Moyen Âge, entre le texte biblique et son commentaire. La nécessité d'accompagner le Livre sacré d'une interprétation autorisée tient au statut de la Bible au sein de la culture chrétienne. Dès l'origine, en effet, le message diffusé par les adeptes du Christ exigea une intense activité de relecture de l'Écriture, destinée à dépouiller l'Ancien Testament de son caractère normatif (la Torah comme recueil de lois) et historique (la Bible comme histoire nationale d'Israël), au profit d'interprétations symboliques visant à faire de l'Incarnation le point de convergence de l'histoire du salut, en même temps que la clé de lecture de l'ensemble du texte sacré ${ }^{30}$. Pour donner un exemple médiéval - parmi des milliers - de cet usage chrétien du texte biblique, on peut évoquer ce manuscrit lyonnais du milieu du $\mathrm{IX}^{\mathrm{e}}$ siècle, dans lequel le texte du Deutéronome est entouré de brèves notes marginales affirmant que le «deutéronome», c'est-à-dire la «seconde loi », renvoie en réalité à la loi chrétienne: «Deutéronome signifie renouvellement de la loi; ce renouvellement s'applique au Nouveau Testament ${ }^{31} »$. On ne pouvait plus clairement vider de sa substance la loi juive. Voilé, le texte biblique appelait ainsi nécessairement les commentaires qui en éclairaient le sens.

Si l'interprétation symbolique de la Bible fut l'œuvre de savants, maitres dans la pratique de l'écriture, dans l'art de l'argumentation et de l'exégèse, elle poursuivait des buts pastoraux et s'adressait au plus grand nombre: il fallut tout d'abord convaincre et convertir à la foi chrétienne, puis imposer et entretenir parmi les fidèles une lecture orthodoxe du texte sacré. Dès lors, tandis qu'une part notable de l'activité intellectuelle fut consacrée au dévoilement de la signification « profonde » et « véritable » de la Bible ${ }^{32}$, le texte scripturaire se trouvait accompagné - et matériellement entrecoupé - de longs commentaires, au sein de recueils tels que les homéliaires, qui servaient tout à la fois de livres de lecture, de méditation et de prédication ${ }^{33}$. Puis, tandis que la glose se substituait au commentaire, de nouvelles formes de prédication, ad populum, se développèrent, toujours à partir du texte scripturaire, dont un verset constituait le thema à partir duquel était bâti le sermon ${ }^{34}$. L'imbrication du texte sacré et de son interprétation fut en quelque 
sorte consacrée, dans la seconde moitié du XII ${ }^{e}$ siècle, par le pape Alexandre III, qui prescrivit que la lecture de la Bible ne se fit plus sine glossa, "sans glose ». Aux XII et XIII siècles, les Bibles glosées, de plus en plus "standardisées", ainsi que toute une série d'instruments exégétiques nouveaux, comme les recueils de distinctiones et les concordances, constituaient des outils indispensables pour les prédicateurs.

Par ailleurs, nous avons vu qu'en raison de la forte coloration ecclésiastique de la pratique de l'écrit, celle-ci se trouva associée, dans le système de représentation dominant, à l'Écriture Sainte, c'est-à-dire au texte par excellence qui faisait l'objet de copies, de transmission, de lectures ${ }^{35}$. Or l'idée que les clercs se faisaient de la maîtrise de l'écriture incluait l'effort de décryptage et d'élucidation du message divin, soit la quête d'un « sens » qui ne pouvait être que celui des « divines Écritures ». Tels étaient, en effet, à partir des $\mathrm{VIII}^{\mathrm{e}}$ et $\mathrm{IX}^{\mathrm{e}}$ siècles, les mots récités au moment de la bénédiction des scriptoria, ces lieux où les moines s'adonnaient à la copie des manuscrits, dans les établissements religieux : «Prière récitée dans le scriptorium. Daigne bénir, Seigneur, le scriptorium de tes serviteurs que voici, ainsi que tous ceux qui y demeurent, afin qu'ils saisissent le sens de tout passage des divines Écritures lu ou copié par eux, et afin qu'ils mènent à bien leur tâche ${ }^{36}$. »

11 Plusieurs historiens, au premier rang desquels il faut mentionner Gilbert Dahan, se sont efforcés de saisir les mécanismes du travail d'exploration du sens mené par les exégètes. En tant qu'ils fondaient des interprétations quasiment infinies du Livre sacré, les commentaires et les gloses du Moyen Âge s'apparentaient aux exégèses traditionnelles. Un certain nombre de caractéristiques sont du reste communes aux exégèses juive et chrétienne, comme la reconnaissance d'une lecture plurielle et presque sans fin du texte sacré, conséquence de la translatio de la parole de Dieu en un langage humain, ainsi que la perméabilité entre l'univers créé et l'Écriture Sainte. Mais les caractères communs ne doivent pas masquer ce qui fait toute la particularité de l'entreprise chrétienne: la nécessité de reconnaître un double niveau d'interprétation de la Bible, «littéral » et «spirituel ». Dans sa somme sur l'exégèse chrétienne en Occident médiéval, G. Dahan tente d'expliquer la nature profonde de ce qu'il appelle le "saut exégétique », ou « saut herméneutique », qui faisait passer toute interprétation du texte biblique du niveau de la "lettre " à celui de l'« esprit " ${ }^{37}$ - selon une lecture allégorique très différente des principes à l'œuvre dans les exégèses juive et musulmane ${ }^{38}$. S'appuyant sur les explications des clercs eux-mêmes, G. Dahan avance que l'originalité de cette exégèse tient au fait que non seulement les mots du texte sacré y sont porteurs de sens (comme l'étaient, dans la culture antique, les mots des textes profanes), mais aussi les réalités désignées par ces mots. Cette distinction entre le sens des mots et le sens des réalités décrites par ces mots permet en tout cas d'entrevoir la complexité des mécanismes de production du sens à l'œuvre dans la pratique exégétique des clercs.

Du point de vue idéologique, le principe de l'interprétation à deux niveaux résultait de la nécessité d'établir correspondances et hiérarchie entre l'Ancien et le Nouveau Testament ${ }^{39}$, mais également, sur le même modèle, entre le passé biblique et la situation présente de l'Église : les faits mis en scène dans les livres vétéro-testamentaires devaient être perçus par les chrétiens, selon une clé de lecture spirituelle, comme l'annonce des réalités de l'Incarnation, ainsi que l'annonce des principes au fondement de l'Ecclesia ${ }^{40}$. L'ignorance du mode de lecture de la Bible fondé sur le va-et-vient entre la lettre et l'esprit fut d'ailleurs dénoncée comme une menace pour l'Église. «Ô, vous les hérétiques, que la lettre tue !» (cf. 2 Cor 3,6$)$, s'exclament, entre la fin $\mathrm{du}_{\mathrm{XII}}{ }^{\mathrm{e}}$ et le début du XIII ${ }^{\mathrm{e}}$ siècle, des 
polémistes reprochant aux "hérétiques» de n'accepter que le seul Évangile, de le comprendre mal en ne le lisant qu'à la lettre et en rejetant les "auteurs modernes", c'est-à-dire les exégètes capables d'appréhender son sens spirituel ${ }^{41}$. Des clercs accusèrent pareillement les juifs - juifs plus ou moins imaginaires, comme l'étaient les hérétiques - de repousser toute lecture allégorique de la Bible ${ }^{42}$.

Le mode de lecture orthodoxe, qui faisait donc appel à un double registre, littéral et spirituel, fut théorisé tout au long du Moyen Âge au sein de traités d'herméneutique où se trouvaient décomposés les ressorts de l'interprétation chrétienne ${ }^{43}$. Au fil du temps, les clercs multiplièrent les catégories propres à rendre compte de la complexité de l'exégèse biblique, ainsi qu'en témoignent les fameux «quatre sens " de l'Écriture définis par les savants du XIII ${ }^{\mathrm{e}}$ siècle et naguère étudiés par Henri de Lubac : à côté du sens « littéral » ou «historique» (qui pouvait comprendre plusieurs niveaux et même inclure un sens figuré), le sens spirituel se subdivisait, en effet, en sens " allégorique », " anagogique » et « eschatologique " ${ }^{44}$. Il semble bien que ce genre de conceptualisation et d'explicitation des règles de l'herméneutique sacrée soit propre à l'exégèse chrétienne.

En 1938, dans un petit livre lumineux intitulé Figura, Erich Auerbach a montré ce qui éloignait cette exégèse d'autres types de commentaire allégorique que l'on rencontre dans nombre de cultures, en particulier dans les mondes grec et romain ${ }^{45}$. Figura, car pour les auteurs chrétiens de l'Antiquité, puis pour les clercs du Moyen Âge, les réalités mises en scène dans le discours biblique "figuraient ", c'est-à-dire prophétisaient ou anticipaient d'autres réalités - parfois bibliques, renvoyant aussi à l'histoire de l'Église ou à la situation présente - sans que la consistance historique des réalités premières fût en rien amoindrie par une telle interprétation figurative : en effet, écrit Auerbach, «la figura est une chose réelle, historique, qui représente et annonce une autre chose tout aussi réelle et historique ${ }^{46} »$. Ainsi le jeu de correspondances et de renvois entre le littéral et le spirituel se doublait-il d'une historicité à plusieurs niveaux, selon laquelle passé, présent et futur se faisaient écho, se répétaient, interféraient.

Il nous a paru utile d'examiner, dans le cadre de ce numéro de Médiévales, quelques domaines ou objets d'interprétation où se serait appliquée une démarche similaire à celle qu'exigeait la lecture de la Bible. C'est ainsi que Bénédicte Sère, Géraldine Veysseyre et Claire Wille ont entrepris d'observer les procédés d'emprunt, de transfert ou de réemploi des formes et méthodes de l'exégèse biblique dans les commentaires philosophiques et les textes de fiction. Ainsi que le montre Bénédicte Sère, le corpus aristotélicien fut étudié à l'aide de techniques et selon des principes herméneutiques analogues à ceux auxquels recouraient les exégètes du texte sacré; il se produisit même parfois de véritables échanges entre l'autorité biblique et l'autorité aristotélicienne, allant, dans l'œuvre du franciscain Guiral ot, jusqu'à une imbrication des deux exégèses. Jamais, cependant, les commentateurs ne distinguèrent dans le texte d'Aristote les deux, trois ou quatre niveaux de sens qui constituaient la clé de l'exégèse chrétienne de la Bible: cette différence essentielle dans le traitement de l'autorité parait confirmer la matrice biblique de la Weltanschauung médiévale fondée sur la pluralité des niveaux de sens. De leur côté, les œuvres « littéraires » du Moyen Âge renvoient à des processus d'écriture et de quête du sens qui ne sont pas sans analogie avec ceux qui caractérisent l'entreprise de décryptage du texte biblique ${ }^{47}$. Certaines de ces œuvres furent d'ailleurs glosées, à l'image du texte sacré. Présenté par Géraldine Veysseyre et Claire Wille, le dossier des gloses sur les Prophéties de Merlin - un texte dont le genre n'est pas sans évoquer celui de plusieurs livres bibliques - atteste bien la mise en œuvre de méthodes de travail similaires à celles 
des exégètes de la Bible (allant jusqu'au recours à des techniques de mise en page identiques). Le cas des gloses d'Alain d'Auxerre, certes exceptionnel et tranchant avec le reste du corpus, paraît indiquer une sorte de contamination des gloses sur les Prophéties de Merlin par le système des gloses bibliques.

L'iconographie médiévale constitue un autre champ que nous aurions dû explorer dans cette perspective. Tout d'abord, Emmanuel Bain y fait allusion dans sa contribution, parce que la plupart des peintures et sculptures mettant en scène des personnages ou des récits bibliques donnent à voir, plus que les figures ou les épisodes présentés dans le texte sacré, les interprétations qui en ont été données par les maîtres de l'exégèse chrétienne ${ }^{48}$. Ensuite, parce que les images elles-mêmes se prêtaient au jeu des lectures multiples, les clercs voyant dans les images le même type d'enchaînement de sens que celui qu'ils observaient dans le texte sacré. Il y avait ainsi de profondes affinités entre le fonctionnement des images et l'intense production exégétique des clercs. Dans les deux cas, ces derniers devaient reconnaître « une superposition de significations, permettant d'articuler différents niveaux de réalité, de façon à s'élever des apparences sensibles jusqu'aux vérités les plus spirituelles». Les images médiévales n'étaient donc pas de vaines apparences, mais, comme l'a soutenu Jérôme Baschet, « des figures dans un monde qui est tout entier figure " ${ }^{49}$. Enfin, des images participaient quelquefois à l'entreprise exégétique : l'interprétation du texte sacré pouvait, en effet, s'appuyer sur l'élaboration d'une image picturale ou d'un diagramme qui représentait tout à la fois un moyen mnémotechnique et un objet de contemplation. Au XII siècle, l'école parisienne de SaintVictor joua ainsi un rôle important dans la mise au point de véritables méthodes d'exégèse visuelle ${ }^{50}$.

17 L'exégèse biblique paraît bien avoir constitué, en Occident, le principal atelier d'interprétation et de production du sens ${ }^{51}$. Peut-être faut-il aller au-delà de ce constat et se demander si l'image de la réalité qu'elle induisait - ce qu'Auerbach appelait « l'étrangeté de la conception médiévale du réel » - ne doit pas être envisagée comme une sorte d'habitus, que l'on qualifiera de figurisme généralisé, dont le travail des exégètes serait alors le paradigme. Les principes à l'œuvre dans les commentaires et les gloses bibliques ne relèveraient donc pas seulement d'une herméneutique au sens technique du terme, c'est-à-dire d'un outil intellectuel acquis dans les écoles, mais également d'une sémantique plus diffuse dans la société, en vertu de laquelle toute réalité renvoyait à une réalité d'une autre nature qui lui correspondait. Si les travaux réunis dans ce volume portent, d'une part, sur les usages sociaux de la Bible, et d'autre part, sur le champ d'extension des techniques exégétiques, il convient d'articuler étroitement ces deux approches, qui gagnent beaucoup à être envisagées comme deux versants de la même question. On trouvera dans les pages qui suivent quelques éléments allant dans cette direction. 


\section{NOTES}

1.Les travaux ont pris la forme d'un atelier organisé par le Centre d'Études Médiévales d'Auxerre, en collaboration avec le CÉPAM (UMR 6130, Université de Nice-Sophia Antipolis/CNRS).

2.G. LOBRICHON, « Usages de la Bible », dans ID., La Bible au Moyen Âge, Paris, 2003, p. 28-54 (ici p. 38).

3. « Capitula Caroli imperatoris et glose in genesim et exodem et leviticum et numerum et in deuteronomium et iudicum et Jesue et Ruth et regum, medium librum in uolumine I ", cité par M. DE JONG, « Monastic Writing and Carolingian Court Audiences : Some Evidence from Biblical Commentary ", dans Le scritture dai monasteri, F. DE RUBEIS, W. POHL éd., Rome, 2003, p. 179-195 (ici p. 195).

4.Parmi de nombreux titres : I. WooD, «Incest, Law and the Bible in Sixth-Century Gaul », Early Medieval Europe, 7/3, 1998, p. 291-304 ; A. FIREY, «Lawyers and Wisdom: The Use of the Bible in the Pseudo-Isidorian Forged Decretals ", dans The Study of the Bible in the Carolingian Era, C. CHAZELLE, B. vAN NAME EDWARDS éd., Turnhout, 2003, p. 189-214.

5.J. BARRAU, « La conuersio de Jean de Salisbury : la Bible au service de Thomas Becket? ", Cahiers de Civilisation Médiévale, 50, 2007, p. 229-244 (ici p. 242).

6.Une étude de cas : Ch. DE MIRAMON, « Déconstruction et reconstruction du tabou de la femme menstruée (XII ${ }^{\mathrm{e}} \mathrm{XIII}{ }^{\mathrm{e}}$ siècle) », dans Kontinuitäten und Zäsuren in der Europäischen Rechtsgeschichte, A. THIER, G. PFEIFER et P. GRZIMEK éd., Francfort-Berlin-Berne, 1999, p. 79-107.

7.G. LE BRAS, « Les Écritures dans le Décret de Gratien », Zeitschrift der Savigny-Stiftung für Rechtsgeschichte. Kanonistische Abteilung, 27, 1938, p. 47-80 ; B.C. BRASINGTON, « Non imitanda sed ueneranda. The Dilemma of Sacred Precedent in Twelfth-Century Canon Law ", Viator, 23, 1992, p. 135-165.

8.J.J. CONTRENI, par exemple, note, à propos de la Bible, « that the culture of one book was really the culture of many books » : «Glossing the Bible in the early Middle Ages : Theodore and Hadrian of Canterbury and John Scottus Eriugena ", dans The Study of the Bible in the Carolingian Era, op. cit., n. 4, p. 19-38 (ici p. 28).

9.Comme aime à le rappeler fréquemment G. LOBRICHON, La Bible au Moyen Âge, op. cit., n. 1, passim. En soulignant la variété des interprétations cléricales et l'absence de tout monolithisme ecclésial, Ph. Buc (cf. ci-dessous n. 22) entend également placer la ligne de fracture ailleurs qu'entre les clercs et les laïcs. Le pape Grégoire VII explique tout de même qu'il a plu à Dieu " que l'Écriture sacrée soit cachée dans certains endroits » (ou « en certains passages »), « de crainte que si elle apparaissait accessible à tous, elle ne soit vulgarisée et mal traitée, et n'induise en erreur les esprits médiocres qui l'auraient mal comprise » (Reg. Greg. VII, MGH Epp. Sel., 2, p. 474.

10.J. GOoDY, La Logique de l'écriture. Aux origines des sociétés humaines, trad. française, Paris, 1986, p. 28-29.

11.AUGUSTIN, De doctrina christiana, proemium 8.

12.M. BLOCH, «La vie d'outre-tombe du roi Salomon », Revue belge de philologie et d'histoire, 4, 1925 (repris dans ID., Mélanges historiques, II, Paris, 1963, p. 920-938). 
13.Évidemment anachronique, la notion de «politique » est en particulier utilisée dans le monde anglo-saxon (cf. ci-dessous n. 19 et 22).

14.W. ULLMANN, "The Bible and the Principles of Government in the Middle Ages », dans La Bibbia nell'alto medioevo. Settimane di studio del Centro italiano di studi sull'alto medioevo, 10, Spolète, 1963, p. 181-227.

15.Pour ne citer que l'une des dernières études sur ce thème : I. ROSÉ, « Le roi Josias dans l'ecclésiologie politique du haut Moyen Âge », dans Mélanges de l'École française de Rome, Moyen Âge, 115/2, 2003, p. 683-709.

16.M.M. GORMAN, Biblical Commentaries from the Early Middle Ages, Florence, 2002 ; M. DE JONG, «Exegesis for an Empress », dans Medieval Transformations. Texts, Power and Gifts in Context, E. COHEN, M. DE JONG éd., Leyde-Cologne-Boston, 2001, p. 69-100; M. LAUWERS, « Le glaive et la parole : Alcuin, Charlemagne et le modèle du rex praedicator. Notes d'ecclésiologie carolingienne », Annales de Bretagne et des Pays de l'Ouest, 111/3, 2004, p. 221-244.

17.Sur l'étude et l'usage de la Bible comme pierre angulaire de la réforme à partir de la fin du viII siècle : C. ChAZELle, B. VAN NAME EDWARDS, «Introduction : the Study of the Bible and Carolingian Culture » dans The Study of the Bible in the Carolingian Era, op. cit., n. 4, p. 1-16.

18.S. DE GREGORIO, « Bede the Monk as Exegete : Evidence from the Commentary on EzraNehemiah ", Revue bénédictine, 115/2, p. 343-369 ; ID., "Bede's In Ezram et Neemiam and the Reform of the Northumbrian Church", Speculum, 79, 2004, p. 1-25 ; ID., Bede, On Ezra and Nehemiah, Liverpool, 2006.

19.Voir par exemple les contributions réunies sous le titre The Power of the Word: The Influence of the Bible on Early Medieval Politics, Early Medieval Europe, 7/3, 1998.

20.Cf. M. DE JONG, « Old law and new-found power : Hrabanus Maurus and the Old Testament ", dans Centers of Learning. Learning and Location in pre-Modern Europe and the Near East, J.W. DRIJVERS, A.A. MACDONALD éd., Leyde-New York-Cologne, 1995, p. 161-176 ; EAD., «The Empire as Ecclesia: Hrabanus Maurus and biblical historia for rulers ", dans The Uses of the Past in the Early Middle Ages, Y. HEN, M. INNES éd., Cambridge, 2000, p. 191-226. 21.E. ORTIGUES, « Haymon d'Auxerre, théoricien des trois ordres ", dans L'École carolingienne d'Auxerre. De Murethach à Remi, 830-908, D. IOGNA-PRAT, C. JEUDY, G. LOBRICHON, éd., Paris, 1991, p. 181-227 (repris dans ID., La Révélation et le droit, Paris, 2007, p. 77-130). Ces trois ordres se substituent (ou se rajoutent) à l'ancienne tripartition sociale des " recteurs », « continents » et " conjoints », qui résultait elle-même d'un montage de nature exégétique entre une prophétie d'Ézéchiel et les trois figures vétérotestamentaires de Noé, Daniel et Job.

22.G. LOBRICHON, «Conserver, réformer, transformer le monde ? Les manipulations de l'Apocalypse au Moyen Âge central ", dans The Role of the Book in Medieval Culture. P. GANZ éd., Turnhout, 1986, p. 75-94 ; ID., «L'ordre de ce temps et les désordres de la fin. Apocalypse et société, du IX ${ }^{\mathrm{e}}$ à la fin du XI $\mathrm{e}^{\mathrm{e}}$ siècle », dans The Use and Abuse of Eschatology in the Middle Ages, W. VERBEKE, D. VERHELST, A. WELKENHUYSEN éd., Louvain, 1988, p. 221-241 [repris dans G. LOBRICHON, La Bible au Moyen Âge, op.cit., n. 1, p. 109-128, 129-144] ; Ph. BUC, L'Ambiguité du Livre. Prince, pouvoir et peuple dans les commentaires de la Bible au Moyen Âge, Paris, 1994. Dans son approche de l'exégèse, $\mathrm{Ph}$. Buc reconnaît une dette à l'égard de $\mathrm{G}$. CASPARY, Politics and Exegesis. Origen and the Two Swords, Berkeley, 1979.

23.I.S. ROBINSON, Authority and Resistance in the Investiture Contest : The Polemical Literature of the Late Eleventh Century, Manchester, 1978 ; ID., « The Bible in the Investiture Contest : the 
South German Gregorian Circle », dans The Bible and Medieval Culture, W. LOURDAUX, D. VERHELST éd., Louvain, 1979, p. 61-84 ; J. LECLERCQ, « Usage et abus de la Bible au temps de la réforme grégorienne ", ibid., p. 87-108. La diffusion et la publicité données au texte biblique avaient jadis été relevées par C. MIRBT, Die Publizistik im Zeitalter Gregors VII., Leipzig, 1894.

24.G. LOBRICHON, « Une nouveauté : les gloses de la Bible », dans Le Moyen Âge et la Bible, P. RICHÉ, G. LOBRICHON dir., Paris, 1984, p. 95-114 (repris dans ID., La Bible au Moyen Âge, op. cit., n. 1, p. 158-172). La technique de la glose n'était pas propre au commentaire de la Bible : A. GRONDEUX, « Auctoritas et glose. Quelle place pour un auteur dans une glose? ", dans Auctor et auctoritas : invention et conformisme dans l'écriture médiévale, M. ZIMMERMANN dir., Paris, 2001, p. 245-254.

25.Les scansions mises en évidence me paraissent en outre correspondre avec celles que je dégage, à partir des interprétations du chapitre 23 de la Genèse, dans Naissance du cimetière, Lieux sacrés et terre des morts dans l'Occident médiéval, Paris, 2005, p. 211-258 (« La sépulture d'Abraham et le champ d'Éphron : modèle scripturaire et pratique sociale »). 26.Voir les remarques de Ph. BUC, L'Ambiguité du Livre. Prince, pouvoir et peuple dans les commentaires de la Bible au Moyen Âge, op. cit., n. 22, p. 40-49 sur la recherche du « bon équilibre » et la mesure des écarts dans la reproduction des textes reçus.

27.Cette « invasion exégétique » est relevée par C. CHAZELLE, B. VAN NAME EDWARDS, "Introduction : the Study of the Bible and Carolingian Culture ", dans The Study of the Bible in the Carolingian Era, op. cit., n. 4, p. 1-16.

28.Une telle définition de l'« autorité » s'inscrit dans un contexte où fleurissaient les polémiques : Auctoritas cereum habet nasum, idest in diuersum potest flecti sensum (ALAIN DE LILLE, De fide catholica, I, 30, PL 210, 333A).

29.La notion de " production du sens » est utilisée ici au sens où l'entend A. GUERREAU, L'Avenir d'un passé incertain. Quelle histoire du Moyen Âge au XXI e siècle ?, Paris, 2001, en particulier p. 233-236. Sur le rôle de l'exégèse biblique dans la production médiévale du sens, voir également F. OHLY, Schriften zur mittelalterlichen Bedeutungsforschung, Darmstadt, 1977 ; Chr. MEIER, « Wendepunkte der Allegorie im Mittelalter : von der

Schrifthermeneutik zur Lebenspratik », dans Neue Richtungen in der hoch- und spätmittelalterlichen Bibelexegese, R.E. LERNER éd., München, 1996, p. 39-64.

30.Sur la mise en place d'un enseignement chrétien fondé sur une relecture du texte sacré : M. DULAEY, Des forêts de symboles. L'initiation chrétienne et la Bible (I ${ }^{e r}-V^{e}$ siècles), Paris, 2001, p. 11-32.

31. "Deuteronomium est ueteris iteratio legis ; quae iteratio ad Nouum pertinet Testamentum »; cf. P.-I. FRANSEN, « Un commentaire marginal lyonnais du Deutéronome du milieu du IX siècle ", Revue bénédictine, 117/1, 2007, p. 31-63, et 117/2, 2007, p. 339-382. 32.On en mesurera l'ampleur en parcourant les 11 volumes du répertoire des commentaires bibliques médiévaux : F. STEGMÜLLER, Repertorium biblicum medii aevi, Madrid, 1950-1980.

33.R. GRÉGOIRE, Les Homéliaires du Moyen Âge, Rome, 1966 ; ID., Homéliaires liturgiques médiévaux, Spolète, 1980.

34. Sur cette pratique de la prédication et sur la place qu'y joua le texte biblique : N. BÉRIOU, L'Avènement des maîtres de la Parole. La prédication à Paris au XIII ${ }^{e}$ siècle, Paris, 1998, 2 vol.

35.Du reste, dans l'Occident médiéval, le mot textus renvoyait au codex et, plus particulièrement, à l'Évangéliaire : A. GUERREAU, « Textus chez les auteurs latins du XII 
siècle ", dans "Textus" im Mittelater. Komponenten und Situationen des Wortgebrauchs im schriftsemantischen Feld, U. KLEINE, L. KUCHENBUCH éd., Göttingen, 2006, p. 149-178.

36." Oratio in scriptorio. Benedicere digneris domine hoc scriptorium famulorum tuorum et omnes habitantes in eo, ut quicquid hic de diuinis scripturis ab eis lectum uel scriptum fuerit sensu capiant, opere perficiant »: J. DESHUSSES, Le Sacramentaire grégorien. Ses principales formes d'après les plus anciens manuscrits, III, Fribourg, 1982, n496, p. 239-245 (ici p. 243) : Orationes ab benedicenda loca regularia in monasteriis. Il s'agit de prières provenant de sacramentaires anciens, notamment le Gélasien du viII ${ }^{\mathrm{e}}$ siècle ; elles arrivent dans les sacramentaires du IX ${ }^{\mathrm{e}}$ siècle par l'intermédiaire du Supplément d'Aniane (CXXXIII. Oratio in scriptorio, $\mathrm{n}^{\circ} 478$, éd. J. DESHUSSES, Le Sacramentaire grégorien. Ses principales formes d'après les plus anciens manuscrits, op. cit., I, p. 481).

37.G. DAHAN, L'Exégèse chrétienne de la Bible en Occident médiéval, XII -XIV siècle, Paris, 1999. 38.G. DAHAN, «L'allégorie dans l'exégèse chrétienne de la Bible au Moyen Âge ", dans Allégorie des poètes, allégorie des philosophes. Études sur la poétique et l'herméneutique de l'allégorie de l'Antiquité à la Réforme, G. DAHAN, R. GOULET dir., Paris, 2005, p. 205-229, qui avance l'hypothèse que l'exégèse juive abandonne l'allégorie pour se tourner vers le mythe (avec le midrash), tandis que l'exégèse chrétienne rejette le mythe (p. 213) ; P. LORY, «Les refus d'une exégèse allégorique du Coran en Islam sunnite », ibid., p. 195-203. 39.Notons que cette attitude idéologique fut favorisée par des réalités d'ordre codicologique : ce type d'attitude par rapport au texte sacré qui supposait de constantes mises en rapport et des jeux de miroir entre les différents livres bibliques, impliquant donc une lecture discontinue, nécessitant des retours en arrière et des sauts en avant, fut certainement favorisée par le passage, entre Antiquité et Moyen Âge, du rouleau (uolumen ) au livre (codex).

40.Sur les relations typologiques ou figuratives entre les faits consignés dans la Bible et les réalités ecclésiales du Moyen Âge : M. LAUWERS, « De l'Église primitive aux lieux de culte. Autorité, lectures et usages du passé de l'église dans l'Occident médiéval (IX ${ }^{\mathrm{e}}-\mathrm{XIII}{ }^{\mathrm{e}}$ siècle) ", dans L'Autorité du passé dans les sociétés médiévales, J.-M. SANSTERRE dir., Rome, 2004, p. 297-323.

41.Sur ces textes, cf. Cl. GOIRAN, Les Écrits antihérétiques, fin XII ${ }^{e}$-début XIII ${ }^{e}$ siècle. L'apport de l'édition de trois traités (Madrid BN 6911, Reims BM 495 et Troyes BM 1068), thèse de doctorat (M. ZERNER dir.), Université de Nice-Sophia-Antipolis, 2007.

42.G. DAHAN, Les Intellectuels chrétiens et les juifs au Moyen Âge, Paris, 1990.

43.Parmi les premiers de ces traités, il faut citer les Formulae spiritalis intelligentiae d'Eucher et le De schematibus et tropis de Bède, puis, au XII ${ }^{\mathrm{e}}$ siècle, le De scripturis et scriptoribus sacris d'Hugues de Saint-Victor. Le nombre des traités du type De expositione sacre Scripture croît alors.

44.Selon la définition « classique » d'Augustin de Dacie : « Littera gesta docet, quid credas allegoria,/Moralis quid agas, quo tendas anagogia. » Cf. H. DE LUBAC, Exégèse médiévale. Les quatre sens de l'écriture, Paris, 1959-1964, 4 vol. ; et dans la perspective de la sémantique (au sens technique du terme) médiévale : L. VALENTE, « Une sémantique particulière : la pluralité des sens dans les Saintes Écritures (XII ${ }^{\mathrm{e}}$ siècle) », dans Sprachtheorien in Spätantike und Mittelalter, S. EBBESEN éd., Tübingen, 1995, p. 12-32.

45.Voir notamment les contributions relatives à l'exégèse antique dans les volumes Allégorie des poètes, allégorie des philosophes..., op. cit., n. 38, et L'Allégorie de l'Antiquité à la Renaissance, B. PÉREZ-JEAN, P. EICHEL-LOJKINE éd., Paris, 2004. 
46.E. AUERBACH, Figura. La Loi juive et la Promesse chrétienne [1938], trad. franç., Paris, 2003 (ici p. 36).

47. Écrire, pour un auteur médiéval, n'est-ce pas avant tout se référer aux réserves d'une tradition dont les textes s'écrivent les uns dans les autres, copies et copies faisant palimpseste et compilation sous la surface de l'écriture actuelle, par où le scripteur relit l'ancien dans le nouveau, et inversement, sans distinction historique? » (R. DRAGONETTI, Le Mirage des sources : l'art du faux dans le roman médiéval, Paris, 1987, p. 41).

48. Maintes études de cas ont récemment montré que l'élaboration des images médiévales, peintes ou sculptées, incarnaient des idées exposées dans les commentaires de l'Écriture et dans la prédication. Ch. FRAÏSSE, « Le cloître de Moissac a-t-il un programme ? ", Cahiers de Civilisation Médiévale, 50, 2007, p. 245-270, dégage ainsi le sens spirituel des sculptures du cloître de Moissac (en 1100), pour le mettre en rapport avec les productions exégétiques du scriptorium de l'abbaye à la fin $\mathrm{du} \mathrm{XI} \mathrm{I}^{\mathrm{e}}$ siècle.

49.J. BASCHET, La Civilisation féodale. De l'an mil à la colonisation de l'Amérique, 3e édit., Paris, 2006, p. 731-739 (ici p. 737 et 739 pour les citations). J. WIRTH, L'Image à l'époque romane, Paris, 1999, p. 379-382, relève toutefois les sémantiques différentes auxquelles renvoient, d'une part, les images carolingiennes et, d'autre part, les images romanes. Voir aussi, sur un autre plan, les remarques de W.J. DIEBOLD, « The New Testament and the Visual Arts in the Carolingian Era, with special reference to the sapiens architectus (I Cor. 3. 10) », dans The Study of the Bible in the Carolingian Era, op. cit., n. 4, p. 141-153, relatives à l'interprétation en termes spirituels de l'architecture et des images matérielles.

50.A. ESMEIJER, Divina quaternitas. A preliminary study in the method and application of visual exegesis, Amsterdam, 1978 ; P. SICARD, Diagrammes médiévaux et exégèse visuelle. Le "Libellus de formatione Arche" de Hugues de Saint-Victor, Turnhout, 1993 (Bibliotheca Victorina, 4) ; D. IOGNA-PRAT, «Édification personnelle et construction ecclésiale », dans L'Individu au Moyen Âge. Individuation et individualisation avant la modernité, B.M. BEDOS-REZAK, D. IOGNA-PRAT dir., Paris, 2005, p. 247-269.

51.A. BOUREAU, La Raison scolastique II, (L'empire du Livre), Paris, 2007, p. 110.

\section{AUTEUR}

\section{MICHEL LAUWERS}

CÉPAM - UMR 6130, Université Nice-Sophia-Antipolis/CNRS, 98 boulevard Édouard Herriot, BP 3209, F-06204 Nice cedex 3 